\title{
Classification and Morphological Extraction of
}

\section{ECG Parameters}

\author{
Phadte Sneha Kisan $^{1}$, Amita Dessai ${ }^{2}$ \\ M.E student, Electronic Communication and Instrumentation, Goa College of Engineering, Farmagudi, India ${ }^{1}$ \\ Assistant Professor, Electronics and Telecommunication, Goa College of Engineering, Farmagudi, India ${ }^{2}$
}

\begin{abstract}
Electrocardiogram (ECG) analysis is used to control and detect cardiac disorders. The amplitude and intervals of ECG control theproper functioning of heart. There is dissimilarity in the extracted features which belong to a normal ECG and irregular ECG hence these extracted features are valuable in ECG arrhythmia detection.Cardiac arrhythmia is a set of conditions in which the heartbeat is irregular, too slow, or too fast.This paper proposes ECG signal classification using anArtificial Neural Network classifier, based on feature extraction of the ECG signal with the help of signal processing algorithms. The management of cardiac disorders is doneby detecting and classifying the different ECG signals with the help of Artificial Neural Network.
\end{abstract}

Keywords: Feature Extraction, Artificial Neural Network, ECG analysis, ECG Morphology, Noise Removal, Arrhythmia.

\section{INTRODUCTION}

Electrocardiogram (ECG) is a diagnosis device which reports the electrical activity of heart. The ECG signal can be measured on the surface of the human body therefore it is called as a noninvasive device. It is the most important data to studycardiac diseases and conditions. ECG is a continuous wave plotted time over the $\mathrm{x}$-axis and $\mathrm{mV}$ on the $\mathrm{y}$-axis. The P-QRS-T waves form one cardiaccycle [1]. This is caused by the repetitive polarization and depolarization taking place to acquire the sufficient flow ofblood throughout the body. The key feature in the ECG is the QRS complex which helps us to differentiate between normal ECG and arrhythmia[2] [3]. Arrhythmias are abnormal heart rhythms [4].Some arrhythmias types are so dangerous that they can cause a heart attack or unexpected death. Consequently automatic Classification and extraction of ECG is vital in the proper management of the heart diseases[5] [6].

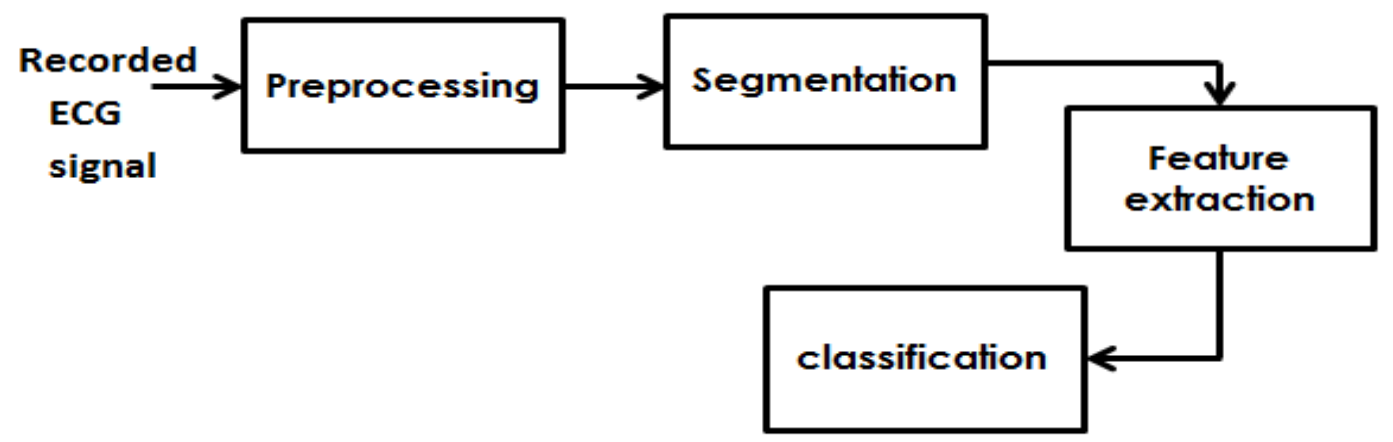

Fig.1. Block diagram of the proposed system

\section{PROPOSED SYSTEM}

The proposed system consists of four steps: step 1, pre-processing of the ECG signals for noisereduction; step 2, segmentation of ECG; step 3, Feature Extraction of ECG; step 4, classification ofECG.

\section{A. MIT BIH Arrhythmia Database}

The ECG signals used in this paper are obtained from the Physionet MIT-BIH Arrhythmia database[7] .The database contains of 48 records. Each of the record is over 30 minutes long. The first set contains 23 records which run from 100 to 124 withparticular numbers missing. This set consists of most common records selected from routine ambulatory practice. The second set contains of 25 records which run from 200 to 234 with certain numbers omitted .This set contains supraventricular arrhythmias, junctional arrhythmias and complex ventricular arrhythmias.Sampling is done at $360 \mathrm{~Hz}$ frequency with the resolution of 11 bits. 
B. Pre-processing of ECG signal

1) Signal Averaging:

Signal averaging is required because two signals are available from the MIT-BIH Arrhythmia database namely modified limb lead II and modified lead V2. Both the signals are obtained by placing the electrodes on the chest. The QRS complex is more prominent in the modified limb lead II signal.

2) Baseline drift filtering:

Baseline drift occurs due to body breathing which causes the ECG waveform wandering.The amplitudes of the ECG waveform changes due to baseline drift [8]. The measuring electrodes impedance is modulated. Baseline drift is removed by subtracting the mean of the signal from signal itself.

3) Powerline noise filtering:

Noise components appear at the powerline frequency $(60 \mathrm{~Hz})$ due to Electromagnetic fields originating from power lines.The interpretation of low amplitude waveforms get mostly affected by these noise components. Power line interference was eliminated by using a notch filter centered at $60 \mathrm{~Hz}$.

4) Low pass and High pass filter:

The low pass filter and high pass filter are designed with the help of Transfer functions. Low frequency and high frequency noises are eliminated with the use of Low pass filter and high pass filter.

The low pass filter (LPF) is represented by the transfer function: $\mathrm{H}(\mathrm{z})=.0002 \frac{\left(1+\mathrm{z}^{-1}\right)^{2}}{\left(1-\mathrm{z}^{-1}\right)^{2}}$.

The high pass filter is represented by the transfer function: $\mathrm{H}(\mathrm{z})=\frac{\left(\mathrm{z}^{-17}-\mathrm{z}^{-16}-.01 \mathrm{z}^{-1}-.01\right)}{\left(-\mathrm{z}^{-1}+1\right)}$

\section{Segmentation}

Segmentation is done for the determination of $\mathrm{R}$ peaks. The following steps are implemented for the determination of $\mathrm{R}$ peaks.

1) Differentiation:

The filtered ECG signal is differentiated to obtain the slope information. Differentiation is done to find the maximum slope of the ECG. QRS complexes have highest slopes. The $\mathrm{P}$ wave and $\mathrm{T}$ wave have small slopes. The $\mathrm{P}$ wave and $\mathrm{T}$ wave which have low frequency components are suppressed by differentiation. It emphasizes the high frequency components of the QRS complex slopes. The derivative is represented by the transfer function:

$y(m T)=0.125 *[-x(-4 T+m T)-2 x(-3 T+m T)+x(m T)+2 x(-T+m T)]$

\section{2) Squaring:}

Squaring is done to make the ECG result non negative. The large differences from the QRS complex, with high frequency are enhanced. The differences of the $\mathrm{P}$ wave and $\mathrm{T}$ waves are compressed which are very small in magnitude.The squaring function is represented by:

$\mathrm{y}(\mathrm{mT})=[\mathrm{x}(\mathrm{mT})]^{2}$

3) Moving Window Integration and thresholding:

The ECG waves contain large amplitude and long duration QRS waves and the slope of R wave cannot properly detect them. A moving window integrator is hence employed to detect these abnormal waves. The difference equation for this moving window integrator is

$\mathrm{y}(\mathrm{mT})=\frac{((\mathrm{x}(\mathrm{mT})-(\mathrm{M}-1) \mathrm{T})+(\mathrm{x}(\mathrm{mT})-(\mathrm{M}-2) \mathrm{T})+\cdots+\mathrm{x}(\mathrm{mT}))}{\mathrm{M}}$

Thresholding is done to detect the $\mathrm{R}$ peaks.

D. Feature Extraction

In feature extraction stage we need to find out the QRS complex onset and offset. Q peak or the S peak is the other maximum slope of the $\mathrm{R}$ wave. In a window backward search is carried out from the $\mathrm{R}$ position. If a zero crossing is detected it is marked as $\mathrm{Q}$ wave. Q wave is said to be lacking if there is no zero crossing inside the window. Forward search from $\mathrm{R}$ position is carried out and the detected zero crossing is marked as QRS offset. S wave is said to be lacking if there is no zero crossing inside the window.

The onset of QRS complex is called as the Q wave and the offset of QRS complex is called as the $\mathrm{S}$ wave.QRS complex is the time period between the $\mathrm{Q}$ wave and the $\mathrm{S}$ wave.From this procedure four features are extracted namely the amplitude of $\mathrm{Q}$ wave, amplitude of $\mathrm{S}$ wave, amplitude of $\mathrm{R}$ wave and QRS complex. 
The fifth feature is the RR interval. It is the distance between two subsequent $\mathrm{R}$ peaks .It is found by calculating the mean of the detected $\mathrm{R}$ peaks. RR intervals also provide us with the heart rate. Heart rate is represented as $=\frac{(60 * \mathrm{Fs})}{(\mathrm{RR} \text { interval })}$ ; where Fs is the sampling rate.

E. Artificial Neural Network

ANN architecture is the interconnection of processing elements called as neurons. The weights define the strengths of these connections. It comprisesof an input neuron layer, output neuron layer and hidden layers.

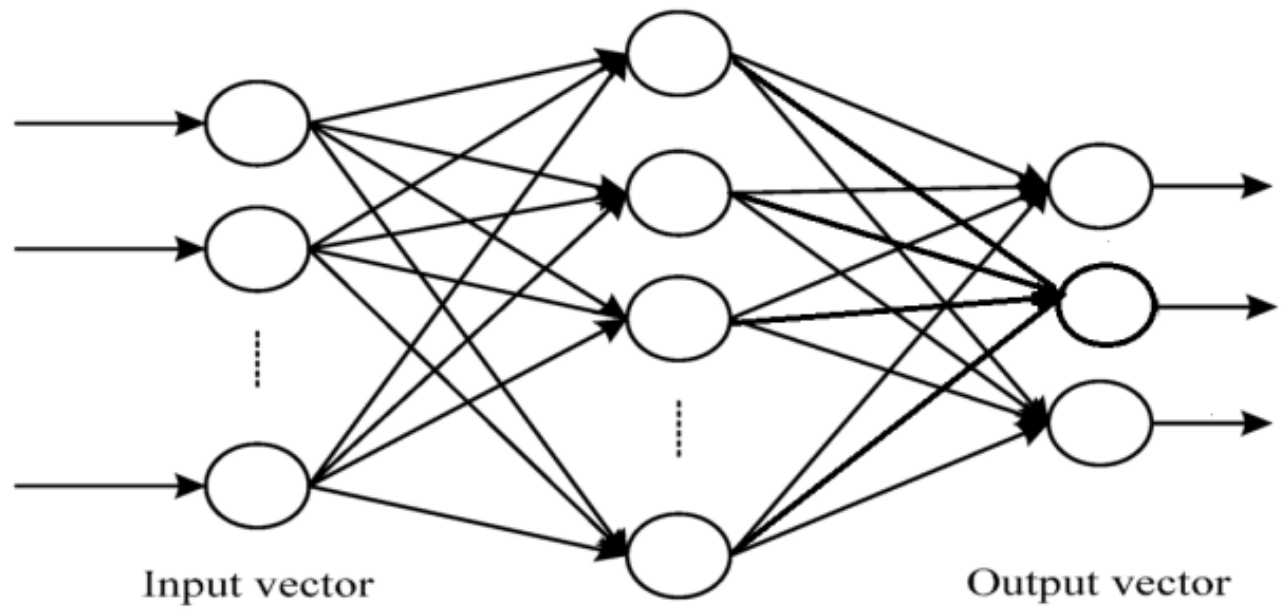

Fig.2. Artificial Neural Network Architecture

The network contains three output neurons and 44 hidden unit neurons. Hidden layers represent the intermediate layerswhich do not mix with outside environment. Trial and error method is used to find the number of hidden layers. The time required to train a network is directly proportional to the number of hidden layers. The neural network uses backpropagation algorithm. Each node of the backpropagation algorithm contains a nonlinear threshold element and hence it is a nonlinear method. The layered structure makes it very complex. Nodes are divided into layers which run from 0 to $\mathrm{L}$. The length of node from the input nodes signifies the layer number. The training function used is traingd; it updates weight and bias values according to gradientdescent. For the proper network development, number of training cycles and network size must be carefully selected. The network does not learn the examples presented to it if the training is inadequate. The network memorizes the training examples if the network is trained excessively. The Artificial neural network classifies the ECG based on the five features into three different classes namely Normal Sinus Rhythm, ventricular tachycardia,Sinus Arrhythmia.

III. RESULTS

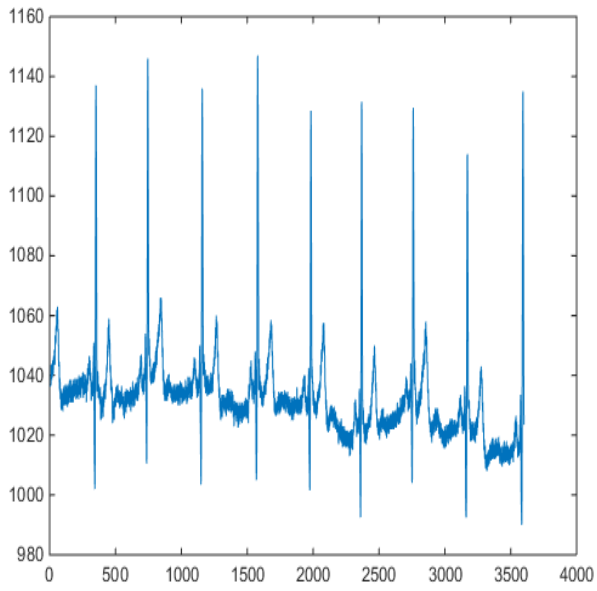

Fig.3. Averaged ECG signal

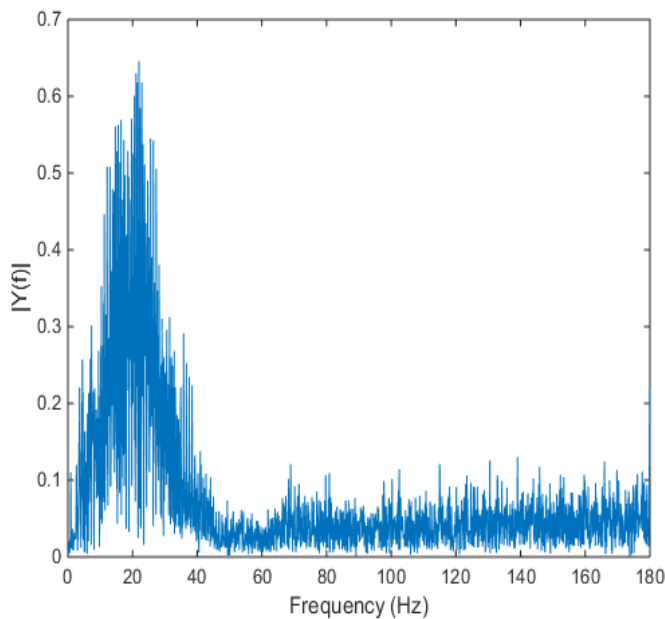

Fig.4. Pre-processed ECG signal in frequency domain 

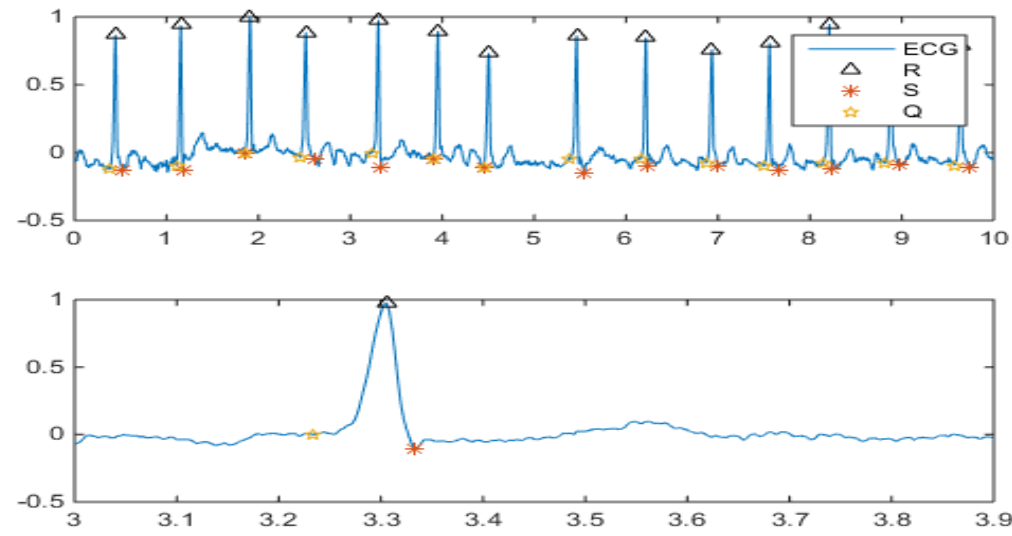

Fig.5. Detected Q peak, R peak and S peak after Segmentation and Feature Extraction of ECG signal
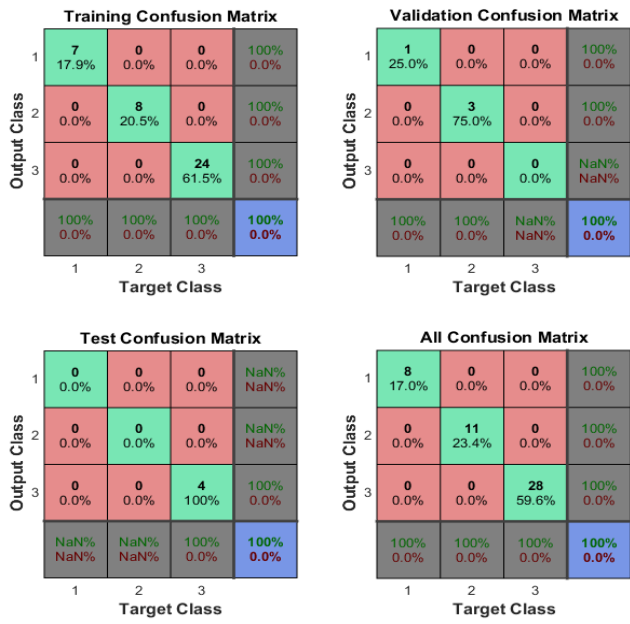

Fig.6.Confusion plot of Artificial Neural Network

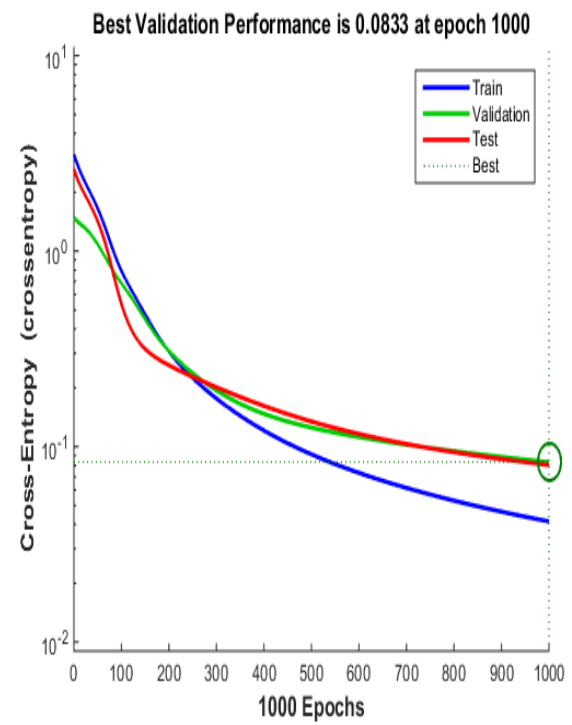

Fig.7. Performance plot of Artificial Neural Network

\section{CONCLUSION}

The Morphological features of the ECG signal are extracted from the proposed system. Features like amplitude of Q wave, amplitude of S wave, amplitude of R wave, QRS complex and RR intervals are used to differentiate between the various ECG signals. The procedure is implemented on MATLAB platform with the intension to extract the ECG features in best possible way.

\section{REFERENCES}

[1]. S.Karpagachelvi, Dr.M.Arthanari, M.Sivakumar,” ECG Feature Extraction Techniques ”, International Journal of Computer Science and Information Security,Vol. 8, No. 1, April 2010.

[2]. Rameshwari S Mane, A N Cheeran, Vaibhav D Awandekar and PriyaRani,"Cardiac Arrhythmia Detection By ECG Feature Extraction", International Journal of Engineering Research and Applications: 2248-9622, Vol. 3, Issue 2, March -April 2013.

[3]. B. Anuradha, V. C. VeeraReddy,"ANN for Classification of Cardiac Arrhythmias”,ARPN Journal of Engineering and Applied Sciences , ISSN 1819-6608, Vol. 3, NO. 3, June 2008.

[4]. Anand Kumar Joshi, ArunTomar, MangeshTomar," A Review Paper on Analysis of Electrocardiograph (ECG) Signal for the Detection of Arrhythmia Abnormalities", International Journal of Advanced Research in Electrical, Electronics and Instrumentation Engineering, ISSN (Online): 2278 - 8875, Vol. 3, Issue 10, October 2014

[5]. A.D.Dongare, R.R.Kharde, AmitD.Kachare," Introduction to Artificial Neural Network", International Journal of Engineering and Innovative Technology:2277-3754, Volume 2, Issue 1, July 2012.

[6]. V.X. Afonso, W.J. Tompkins, T.Q. Nguyen, and S. Luo, "ECG beat detection using filter banks", IEEE Trans. Biomed. Eng., vol. 46, pp.192202, 1999.

[7]. MIT-BIH Arrhythmia Database (Cited in 8 Octomber 2015): http://physionet.org/physiobank/database/mitdb/

[8]. Rajesh D. Wagh, Kiran R. Khandarkar, Dipanjali D. Shipne, Shaila P. Kharde,” Noise Removal from Electrocardiogram (ECG) a Comparison Approaches", International Journal of Advanced Research in Computer Engineering \& Technology (IJARCET) Volume 3 Issue 1, January 2014. 